\title{
La articulación de ámbitos sociales a partir de la base temporal
}

\section{Carlos Lozares \\ Pedro López}

Universitat Autònoma de Barcelona. D epartament de Sociologia

08193 Bellaterra (Barcelona). Spain

\section{M àrius D omínguez}

U niversitat de Barcelona. D epartament de Sociologia i M etodologia de les Ciències Socials 08034 Barcelona. Spain

\section{Resumen}

Uno de los objetivos de la investigación ha consistido, en un primer nivel de análisis, en conseguir una tipología general de la forma de vinculación entre los ámbitos de TP (trabajo productivo), TR (trabajo reproductivo) y TL (disponibilidad de tiempo libre) a partir de datos e indicaciones cuantitativas. D espués de diversos ensayos, los resultados y el procedimiento más simple y eficaz se han conseguido a partir de dimensiones indicativas del empleo del tiempo para cada uno de los ámbitos. Por tanto, una vez construidas las tipologías de cada uno de los ámbitos, se toma el tiempo, en este artículo, como referente 0 sustrato para la constitución de modos de vida cotidiana. Los tipos de modos de vida cotidiana serán a su vez la base de la intervención por entrevistas en la segunda y tercera parte de la investigación. El artículo muestra cómo el tiempo, en tanto que instrumento y soporte para la construcción de los modos de vida, pasa a ser un descritor y clasificador de grupos sin necesidad de recurrir directamente a las variables categoriales: sexo, categorías sociales, edad, etc.

Este artículo es parte de una investigación cuyos resultados aparecen en este mismo número de PAPERS. El artículo tiene autonomía y entidad por sí mismo pero la lectura del resto de los artículos de este número facilitará su contextualización.

Palabras clave: tiempos sociales, vida cotidiana, grupos sociales.

\section{Abstract}

In an initial level of analysis, one of the objectives of the research project consisted in obtaining a general typologie of the way in which the results obtained in each area, PW (Productive W ork), RW (Reproductive Work) and FT (Availability for Free Time) were linked and articulated using quantitative data and indications. After several trials the results and the simplest and most effective procedure were obtained from indicative dimensions of the use of time for each area. Therefore, once the typologies of each area had been constructed, in this article time was taken as a referent or substratum for the constitution of D aily Ways of Life, that is to say, as a criterion for articulating the initial Typologies. The Types of $D$ aily Ways of Life will in turn be the basis for action through interviews in the second and third part of the research. The article shows how time as an instrument and a support for the construction of ways of life becomes a social describer and classifier of groups without the need for direct use of categorial variables: sex, social categories, age, etc. 
This paper is part of a research work whose results are in this PAPERS volume. The paper is autonomous but the reading of the other papers of this volum can help to contextualize it.

Key words: social times, everyday life, social groups.

\section{Sumario}

1. La problemática y el marco teórico

2. Objetivos y supuestos de los análisis presentados referentes a los tiempos sociales v a los tipos de modos de vida cotidiana. El procedimiento
3. Los resultados

4. Reflexiones para unas conclusione

Bibliografía

\section{La problemática y el marco teórico}

\subsection{D e qué se trata}

Las preguntas básicas que nos formulamos en este artículo son las siguientes. ¿Es posible encontrar pautas de comportamientos homogéneos en las formas de articular las actividades de la gente para determinadas secuencias temporales? Esto es, ipueden darse tipos o modos de vida cotidiana, si éstos los entendemos como dichas formas pautadas de desarrollarse las secuencias de las actividades corrientes? Además, ihasta qué punto dichos modos de vida tienen algo que ver con grupos sociales provenientes de las diferencias de género, edad, categoría socioeconómica y otros? ¿Puede darse una lógica social proveniente del desarrollo y encadenamiento de la vida diaria - actividades, tiempos, lugares, relaciones, estrategias, etc. - que dé cuenta directamente de las diferencias sociales, siendo los factores estructurales el marco resultante contextual, o bien es dicho contexto determinante y decisivo sobre las acciones y sus articulaciones cotidianas y que en cierta manera las absorben? ¿C uál es y cómo se realiza el nexo entre ambas lógicas si, como es presumible, se da una correlación entre ambas? U na correspondencia absoluta entre los tipos de modos de vida cotidiana y los grupos sociales abundaría en la idea o hipótesis de su equival ecia y lógicamente de su mutua determinación. Si así fuera, el tiempo con el espacio y las relaciones que son tomados como soporte de la articulación de las actividades, y evidentemente ellas mismas, podrían llegar a ser, por lo menos, un buen operador de clasificación social con resultados equivalentes a las variables estructurales cuyo poder atributivo en la constitución de la estructura y grupos sociales se da por supuesto. Pero, iy si no se diera tal coherencia o cuasiequivalencia? Pudiera serlo sólo parcialmente o no serlo en absoluto. En este caso, el poder estructurador que tendría el tiempo como soporte social no sería equival ente al de los factores sociales más estructurales. Por lo menos habría que afirmar que para esta situación, o secuencia de situaciones, hay o se da un problema: el fluir de la 
vida cotidiana reflejada en los modos de vida cotidiana, en los tipos de vida cotidiana, no configura unas pautas de vida social equiparables a los provenientes de dinámicas más macrosociológicas propias de la estructura social. ¿La vida cotidiana iría entonces por unos derroteros, al menos para aquella situación o situaciones, diferentes a los grupos sociales macros? ¿Sería, si éste fuera el caso, el inicio de conflictos, disfunciones o contradicciones sociales manifiestos o larvados camino de un nuevo reequilibrio, o de una situación de inesta bilidad? ¿o sería simplemente el signo de la impenetrabilidad o de la resistencia de determinadas situaciones y momentos a la estructura social? N uestro supuesto es que se da un grado elevado de correspondencia entre modos de vida cotidiana y grupos de la estructura social en particular si se contemplan poblaciones extensas y secuencias y espacios amplios. Precisamente la investigación que presentamos va orientada a esta afirmación.

Y por fin, ¿cómo elaborar, metodológicamente hablando, dichos modos de vida «desvinculados o desguarnecidos» en su construcción del armazón categorial típico de los análisis de estructura social? Es decir, ¿cómo construir unas entidades en las que se manifieste solamente la inercia o dinámica propia de la vida tal y cómo se da?

\subsection{La identidad social del tiempo}

D ado que el tiempo es el sustrato y el elemento conductor de la construcción de los modos de vida cotidianos, comenzaremos con al gunas reflexiones generales sobre el mismo.

El tiempo es concreto y es cercano, y al mismo tiempo abstracto y genérico. El tiempo es subjetivo, ligado a las representaciones de nuestras acciones, y es social porque en él y por él se da la interacción entre agentes 0 actores sociales. Además, hay diversos tiempos sociales: unos abocados hacia un objetivo y lineales en su progresión y otros circulares y repetitivos en sus días, meses 0 años o en tiempos de vida; hay tiempos de seguridades y tiempos de incertidumbre, tiempos del trabajo, del ocio y de la reproducción de la existencia, etc., etc.

El tiempo tiene además un componente objetivo pero también lo construimos socialmente. Participa, pues, de esta dualidad permanente propia de todo hecho social. Como realidad objetiva es soporte de actividades; como construcción simbólica es soporte de representaciones sociales. Además es, por este mismo juego dual, productor y resultado de la socialización y juego de intereses sociales.

Además, desde la perspectiva social que lo contemplamos aquí, el tiempo no es un absoluto homogéneo. Es un concreto social en el que se dan texturas objetivas diferentes y construcciones socialmente distribuidas. Es diverso como lo es la sociedad (A. Wallemac, 1988: 229-238). No es el mismo tiempo el de una vida planificada, organizada, inalterable y previsible queel de una vida sobresaltada por lo inesperado o por la superviviencia del día a día; el tiempo no es idéntico en quien dispone de mecanismos de poder para crear acontecimientos que el de la persona que vive al remolque que se le impone; el 
tiempo difiere en quien «dispone» de él por desocupación obligada o en quien está superocupado.

Lo importante, y de ahí viene su definición social, es que el tiempo nos reenvía objetivamente a la actividad o a la práctica y menos a concepciones metafísicas o a un tiempo universal. El tiempo social es la referencia que da cuenta o expresa el cambio o el movimiento de los fenómenos sociales con relación a otros hechos sociales. Su identidad social le viene de que es un referente de los cambios que se dan en los fenómenos sociales. Así, el tiempo no es desvinculable, en nuestra perspectiva, de las prácticas sociales, sean éstas individuales o colectivas y, por tanto, de las actividades de interacción social. En este sentido, son las actividades las que producen el tiempo social. Incluso como construcción simbólica no se trata solamente de una representación 0 interpretación, sino que también interviene como proceso intencional en la práctica real. N . Elias da una definición general y concreta del tiempo: el «tiempo» es «el símbolo de la relación que un grupo humano - es decir los seres biológicos dotados de capacidad de memoria y síntesis- establece entre dos 0 más continuums de cambios, de los cuales uno está utilizado como cuadro de referencia del otro o los otros (N. Elias, 1985; E.R. Leach, 1961: 124-143; M. Aymard, A. Grignon y F. Sabban, 1993: 1-37).

$D$ ado que solamente a partir de la acción y por la acción se originan y manifiestan los cambios sociales, la variabilidad de los hechos pasa a ser el criterio observable para identificar el tiempo social. Además, dado que toda acción social es de interacción, será en y a partir de ella que se construye socialmente el tiempo. Al no ser la interacción desligable de la situación, ésta última se convierte en la unidad más elemental de análisis del tiempo social y éste en patrón de «medición» de la misma situación. Correspondientemente, el cambio de situación pasa a ser el criterio de la variabilidad y la fuente de la identidad social del tiempo.

\subsection{La diversidad de los tiempos sociales}

El tiempo puede ser contemplado con diferentes ritmos, cadencias e interiorizado según diferentes representaciones dada la vinculación que con la acción tienen los distintos actores sociales. Tenemos el «tiempo diario» asentado básicamente sobre el tiempo de sol, del día y de la noche y sobre unos ritmos biológicos de descanso y sueño. Es el tiempo habitualmente llamado «cotidiano» y equiparable con la rutinas. Está muy marcado por las dos actividades, la productiva y la reproductiva: tiempo de trabajo, de acumulación y desgaste, tiempo que asegura recursos y llena necesidades. También puede ser un tiempo de olvido y evasión de otros tiempos. Este tipo de tiempo diario será el objeto directo y exclusivo en nuestro análisis.

Pero podemos hablar también de otros tiempos, por ejemplo de los ritmos «semanales» $y$ «estacionales», que son tiempos de ruptura entre el tiempo productivo y el del ocio; del «tiempo del ciclo de vida» o «tiempo de vida», que se interioriza como biografía y como historia de vida con un sentido específico para 
cada sujeto social vivido como propio e intransferible (C. Lalive d'Epinay, 1988: 15-31; S. Tabboni, 1986: 25-28). Se habla también del «horizonte temporal», es decir, «de una manera de ver, de un ángulo de visión tal que permite elaborar anticipaciones sobre el futuro en términos de proyecto basados en las experiencias individuales y colectivas accesibles por la memoria y teniendo en cuenta los constreñimientos y oportunidades de la situación presentes (R. Rezsohazy, 1988: 181-190). El horizonte temporal es un conjunto de actitudes y representaciones que transcurren entre el pasado, el presente y el futuro permitiendo organizar la experiencia. El «tiempo de vida» y el «horizonte temporal» se constituyen a partir de lo vivido individualmente, pero también por experiencias participadas colectivamente: influyen en el presente tanto como configuran el futuro a través de expectativas y proyectos (M. Wallace, 1977; P. Bourdieu, 1963; P.A. Sorokin, 1936). Podemos hablar también del «tiempo de la historia» como el de un proyecto camino de una diana o de un objetivo social con sentido. Se trata de un tiempo más vinculado a los acontecientos exteriores, al de las creencias y representaciones compartidas en una dinámica col ectiva y está marcado por acontecimientos importantes (C. Lalive d'Epinay, 1988: 15-31).

En el estudio que presentamos ninguna de las conceptualizaciones temporales del párrafo precedente es objeto directo de análisis, no por su justificada pertinencia, sino porque esta investigación parcial se enmarca en unos objetivos que no contemplaban dichas variedades temporales. Así, nos limitaremos a la secuencia temporal del día laborable, más cercana a la idea que se tiene corrientemente de lo cotidiano, y dejando además fuera de nuestra conside ración todo lo que podríamos llamar construcción simbólica del tiempo más vinculada a los conceptos de tiempo de vida, horizonte temporal, tiempo vivido y tiempo de historia. Es evidente que también en el tiempo diario influyen, y de él se nutren, todas estas visiones temporales en forma de representaciones, valores y estructuras de sentido, pero nuestro análisis se centra en una representación del tiempo muy denotada y objetivada a partir de las actividades declaradas por los sujetos examinados.

\subsection{D imensiones que atraviesan el tiempo social}

Las diversas connotaciones temporales precedentes, y otras no mencionadas, se encuentran en la base de las dimensiones que atraviesan el tiempo. Dimensiones que a su vez son buenas guías para operativizarlo. Consideraremos solamente aquéllas que son más pertinentes para los objetivos de nuestro análisis.

Una primera concepción del tiempo lo sitúa en una dimensión que va desde una objetivación y/o interpretación cíclica del mismo hacia un concepción más lineal. El tiempo se puede presentar, por un lado, como un retorno permanente - la noche sucede al día y viceversa, la tarde a la mañana y la noche a la tarde, etc., el invierno al verano: «otro tiempo será», «arrieros somos»-. Las actividades se muestran entonces ligadas o bien a los tiempos biológicos 0 a las estaciones o ritmos diarios, pero también a la pasividad que da la inexorabilidad de un destino que se impone circularmente. Por otro lado, el tiempo 
puede verse como un tiempo lineal, como por ejemplo el tiempo de la planificación estratégica basado en una idea de continuidad, de desarrollo y de los objetivos a corto, medio y largo al cance. Esta segunda dimensión corresponde más propiamente a la sociedad moderna y productiva, a la rígida medición de las actividades por el tiempo, a la visión del pasado como acumulación de experiencias, hechos o bienes pero canalizable y autopropulsado, como carrera y biografía, a un tiempo previsible y organizado.

Paralela a esta distinción es la que considera el tiempo entre, por un lado, vinculado a específicas tranforormaciones cíclicas, de tal forma que la duración de su periodicidad se puede tomar como patrón de las actividades hasta, por otro lado, como un tiempo abstraído de toda eventualidad cíclica y de cualquier manifestación fenomenológica, confiriéndole un estatus de medida universal (M. Aymard, A. Grignon y F. Sabban, 1993: 1-37). Con relación a esta idea aparece también la visión del mismo como un proyecto exógeneo que se nos impone en sus regularidades hasta la idea de un tiempo abierto que da rienda suelta a la libertad del proyecto y de la previsión. Esta dicotomía es paralela y equivalente a la del sujeto social como agente pasivo sometido a lo inexorable, 0 a la del actor como sujeto de proyectos, de estrategias, con capacidad de imaginar el futuro y de actuar sobreél. «Una sociedad no es sólo la estructuración que la determina, es también los desseins que la mueven» (R. Rezsohazy, 1988: 181-190).

Evidentemente, no entra en los objetivos de nuesta investigación tener en cuenta toda esta riqueza connotativa. Solamente consideraremos la periodicidad y linealidad que pueda darse en las temporalidades de mañana, tarde y noche partir de las actividades vinculadas a ellas.

U na segunda dimensión que es interesante para nuestros propósitos es la de continuidad del tiempo opuesta a la idea de fragmentación o discontinuidad del mismo. Por la primera polaridad, el contenido del tiempo es dado y contemplado como una sucesión de actividades y de representaciones en secuencias coherentes y relacionadas que se hilvanan y se comunican su sentido objetivado y/o interpretado. De alguna manera, unos hechos pasan a ser instrumentos para otros que dan o se piensan como objetivos de los primeros. Por contra, en el otro polo, se da y/o experimenta un tiempo más cambiante de rupturas aparentemente incoherentes, lo que no quiere decir que no obedezcan a una lógica más latente o más general (S. Tabboni, 1986: 25-28).

\subsection{Los modos de vida cotidiana y el tiempo social}

La investigación que presentamos se refiere exclusivamente a las actividades realizadas en un día laborable y por tanto a los modos de vida provenientes de dicha secuencia temporal. Sin entrar en excesivas consideraciones sobre lo que se puede entender por modos de vida cotidiana, seguiremos para nuestros propósitos las ideas y orientaciones de Bertaux y Javeau.

«Al menos inicialmente lo esencial de la vida cotidiana, en el pleno sentido del término, está constituido, para el común de los mortales, por prácticas repetitivas, de trabajo, de transporte del domicilio al trabajo y vuelta, de la 
compra de alimentos, de los trabajos en el hogar y por las interacciones "banales" entre los miembros del hogar familiar» (D. Berteaux, 1983: 69). Pero para él la vida cotidiana no se queda ahí. Estas prácticas, en sí aparentemente insignificantes, se estructuran en ciclos: diario, semanal, estacional y anual; de empleo y paro; de enfermedad y salud; de juventud y vejez. Se establecen según estrategias y objetivos familiares, por ejemplo: prevenir riesgos como los créditos; los seguros y el ahorro. Se organizan no sólo sincrónicamente, sino también evolutiva o diacrónicamente. Su síntesis evolutiva constituye lo que se estará tentado en designar como «modos de vida» (D. Berteaux, 1983: 69). Bajo esta idea de modos de vida está implícita la de producción ampliada, ya que para Berteaux la distinción entre reproducción y producción tal y como se entiende carece de sentido. Berteaux denomina a la producción ampliada «ugar de la antroponimia», esto es, «ugar de producción de la energía humana» (D. Berteaux, 1983: 73). Lo que aparece central es «el desarrollo en el tiempo del modo de vida» (D. Berteaux, 1983: 75). Al mismo tiempo, ofrece una orientación metodológica y de contenido para la constitución de tipos de modos de vida. Es evidente, desde nuestra perspectiva, que estos modos de vida no son autónomos e independientes de la realidad social. Como afirma N . Elias, «o cotidiano es un dato societal» que en el análisis no puede separarse «de las estructuras globales del poder».

C. Javeau plantea una construcción de la vida cotidiana, para nosotros modos de vida, en diversos y sucesivos niveles. Veamos lo que más puede interesarnos desde nuestra perspectiva.

«Lo cotidiano hace referencia, primero, a la noción de duración, la de 24 horas, definida materialmente por la astronomía» (C. Javeau, 1980: 32). En esta primera caracterización introduce la dimensión tiempo como factor de corte secuencial. Esta duración se va llenando de contenido y hace que la vida cotidiana se constituya en y de actividades: comer, desplazarse, levantarse, hablar, etc. Los tipos de modos de vida que se construyan tendrán esta base objetivable. Pero la «sociología de la vida cotidiana se interesa por estas actividades (regulares) solamente en la medida en que se realizan de manera relativamente estandarizada, en conformidad con un número limitado de modelos que están en la base de la cultura de un grupo» (C. Javeau, 1980: 34). La vida cotidiana inicial, verdadero cajón de sastre, se «des-amorfa» y se homogeneiza y, modelizándose, permite autoaplicarse a determinados agrupamientos sociales. Es lo que llamaremos «modos o tipos de vida cotidiana», cuya construcción es uno de los objetivos de esta investigación.

El paso siguiente consistirá para Javeau en tomar en consideración el punto de vista de los sujetos identificados: el stock de conocimentos, la identidad de los contenidos, la interacción y la situación. Se trata de una vuelta al sujeto (C. Javeau, 1983: 25). Este nivel no entra directamente en nuestro trabajo, solamente algunos de los aspectos del segundo nivel son tenidos en cuenta.

El tercer elemento consiste en la conexión con la sociedad global. Javeau plantea como problema importante las vinculaciones posibles entre una perspectiva microsociológica, que podría recuperar y comprender los dos niveles 
anteriormente descritos, y lo global social. Para Javeau pueden seguirse varios caminos. En realidad, esta correspondencia o adecuación es uno de nuestros supuestos. Por nuestra parte, una vez constituidos los tipos de modos de vida los «confrontaremos» con las variables clásicas que tienen una función importante en la categorización de la sociedad: categoría socioeconómica (C SE), edad, sexo, tipologías familiares, etc.

\section{0 bjetivos y supuestos de los análisis presentados referentes a los tiempos sociales y a los tipos de modos de vida cotidiana. El procedimiento}

\subsection{Los objetivos}

El primer objetivo que nos marcamos tiene que ver, pues, con la construcción de los tipos de modos de vida cotidiana entendidos como formas homogéneas de articular diversos ámbitos de actividades: en nuestro caso, estos ámbitos son: el de las actividades productivas o tiempo productivo, TP, el de las actividades reproductivas o tiempo reproductivo, TR, y el del tiempo libre, $T L$, durante un día laborable. Al constituir dichos tipos de modos de vida no se hacen intervenir, en una primera instancia, otros contenidos o variables que los referidos; se constituyen pues sin la presencia de otros más estructural es o categoriales. D e esta manera, la tipología o las diversas configuraciones que surjan serán solamente, como anunciábamos, propias y exclusivas de la articulación del fluir de las actividades cotidianas: se trata, por tanto, de esta blecer de manera autónoma los tipos de modos de vida. La confrontación será a posteriori, lo que posibilitará el poder analizar las correspondencias u homologías entre la tipología de los modos de vida encontrados y los grupos provenientes de los factores sociales. En todo ello el tiempo tiene una función de instrumento como soporte y medición de la vinculación de activida des. Aparte de esta función, el tiempo es un hecho social desigualmente distribuido.

\subsection{Los supuestos o hipótesis guía}

Los objetivos planteados suponen una serie de hipótesis que más bien tienen un carácter orientativo y descriptivo. Partimos del hecho de considerar el tiempo como una realidad social, de tal forma que su identidad surge precisamente de la variabilidad de las actividades y relaciones sociales. Evidentemente, estamos interesados no en cualquier tipo de temporalidad sino solamente en la de las 24 horas.

La hipótesis básica consiste en suponer la existencia de formas tipificadas y homogéneas (pautas) de articulación de actividades entre sí. Por tanto, se trata de una hipótesis de existencia de dichos tipos o modos de vida cotidiana. ¿Es homogéneo el día para la gente? ¿Lo es para todos y para todos de la misma manera? ¿Cómo se constituyen estas composiciones temporales a lo largo de 
un día? ¿Se dan rupturas entre ámbitos de vida - producción, reprodución y tiempo libre - o entre la mañana, la tarde y la noche?

En un segundo supuesto pretendemos comprobar hasta qué punto dichas pautas de comportamiento articulado (modos de vida cotidiano) tienen una correspondencia con los clásicos grupos sociales, esto es, que los modos de vida están también connotados socialmente. ¿Cómo se reflejan en ellos los grupos sociales? ¿Q ué es primordial, la categoría social que origina estos tipos y pautas de comportamiento articulado o son dichos tipos los que crean las categorías sociales?

Por supuesto, para analizar todo ello nos quedamos en un primer nivel de tratamiento de los modos de vida, aquél que aparece más centrado en los indicadores, en los aspectos más denotados, y menos en las cuestiones vinculadas al sentido y/o significado dado por los actores. D e alguna manera evacuamos el sujeto como fuente de sentido de la actividad.

\subsection{El procedimiento}

El marco de referencia y los objetivos llenados por la investigación general presentada en este número han quedado suficientemente explicados en los artículos precedentes. La primera fase de esta investigación general consiste en conseguir - llegar a construir tipos o modos de vida cotidiana a partir de la articulación de tres ámbitos: el productivo (TP), el reproductivo (TR) y el del tiempo libre (TL). Previamente, en cada uno de estos ámbitos se ha construido una tipología correspondiente elaborada a partir de conjuntos de indicadores propios de cada uno de dichos ámbitos tomando los datos de una gran encuesta.

El método para llegar a las tipologías de cada ámbito ha sido diverso y acumulativo: tratamientos bivariados, métodos aditivos, log-lineal, análisis de correspondencias. Todo ello ha asegurado la consistencia y validez de las tipologías por ámbito. Una vez conseguidas, se ha tratado de articularlas en una tipología global, es decir, en una tipología general de modos de vida cotidiana como interacción de ámbitos. Esta tipología se ha construido básicamente por procedimientos de log-lineal y no ha sido objeto directo de ninguno de los artículos. Una vez conseguido dicho resultado (modos de vida cotidiana) de la manera descrita la pregunta que nos hicimos fue la siguiente: ino sería posible llegar a resultados similares a partir de una única variable en cada ámbito, por tanto de una manera más directa y simple, sin perder además el objetivo de conseguir los modos de vida cotidiana como articulación de los ámbitos productivo, reproductivo y de tiempo libre?

En la misma encuesta que nos ha servido como fuente inicial de datos, y después de un laborioso camino de reconstrucción, ya que la encuesta no esta ba directamente pensada para ello, hemos llegado a diferenciar y calcular seis variables temporales: «tiempo dedicado a dormir», «tiempo dedicado al trabajo productivo», «tiempo dedicado al trabajo doméstico», «tiempo libre», «tiempo de transporte», «tiempo despierto». Como se ve, el tiempo es un soporte, es una unidad de medición y, a su vez, tiene un contenido y una identidad 
ligados a la actividad que mide: homogeneiza y simplica el proceso sin perder los contenidos sustantivos del objetivo que perseguimos. C ada una de estas variables es además cuantitativa, con lo que potencia el análisis. Además, su conjunto llena la articulación entre los tres ámbitos en los que estamos inte resados - productivo, reproductivo y de tiempo libre - U na vez selecionadas las variables y en vistas a lograr su máxima simplificación y los tipos de modos de vida cotidiana hemos elegido el análisis de los componentes principales, $A C P$, y el cluster AC como los métodos más adecuados para tales objetivos. Veamos rápidamente los resultados.

\section{Los resultados}

3.1. La dimensionalización temporal y la tipología de los modos de vida cotidiana a partir de la articulación de los ámbitos productivo, reproductivo y de tiempo libre

Tres ejes explican la práctica totalidad de la varianza. Una primera tendencia está dada por el tiempo de trabajo versus tiempo libre, esto es, define la relación tiempo productivo/tiempo libre. Ambos ámbitos ocupan por tanto una posición opuesta en la dimensión. U na segunda tendencia está marcada por la dimensión de tiempo doméstico versus tiempo de no dedicación a dicho trabajo. Esta dimensión guarda su autonomía con relación a la primera y al conjunto de las otras variables. Una tercera tendencia viene dada por el eje «biológico» del tiempo de dormir versus tiempo de despierto. Este tiempo apa rece, pues, independiente de los dos primeros ejes. Consideraremos solamente las dos primeras tendencias, ya que acarrean un buen porcentaje de varianza y tienen mayor interés para el objetivo de articular los ámbitos productivo, reproductivo y de tiempo libre.

\subsection{La constitución de los tipos o modos de vida cotidiana}

\subsubsection{El proceso metodológico}

U tilizando como punto de partida las tendencias precedentes se ha procedido, por el Análisis de Cluster (AC), a realizar una clasificación de los individuos en tipos en función de la discriminación que los propios ejes procuran. Se han tomado solamente los dos primeros ejes. Se han ensayado diversos clusters. Inicialmente uno que nos generaba cinco tipos pero aglutinaba casi toda la población en un tipo medio que satelizaba a pequeños grupos en el resto. Al pasar a seis se lograba precisamente esta partición de la media, con lo que los resultados quedaban equilibrados.

\subsubsection{Los resultados}

Tipo 1: Está caracterizado por disponer de bastante tiempo libre, 10 horas diarias; tiene poca dedicación al trabajo productivo, 0,1 horas diarias. Es un grupo relativamente compacto con relación al primer eje. Está también carac- 
terizado por dedicar un buen tiempo al trabajo doméstico. Está en el cuadrante superior izquierdo de los ejes. Reúne a más de un cuarto de la población ${ }^{1}$.

Tipo 2: Está caracterizado por la dedicación al trabajo productivo (8 horas diarias) y por disponer de un tiempo libre de 7 horas diarias, además de no realizar trabajo doméstico. Como se observa por su posición es simétrico radialmente al precedente. Se encuentra en el cuadrante inferior derecho de los ejes. Contiene más de un tercio de la población. Entre los dos primeros forman los dos tercios de la misma².

Tipo 3: Está caracterizado simultáneamente por su dedicación al trabajo productivo, 8 horas diarias, su poco tiempo libre, 3 horas diarias, y su elevado tiempo dedicado al trabajo doméstico. Se sitúan en el cuadrante superior dere cho opuestos, sobre el eje horizontal, a los grupos 1 y 4 y al 2 por el eje vertical. Llegan casi al $10 \%$ de la población 3 .

Tipo 4: Está caracterizado por ser un grupo muy compacto en los dos ejes. Se trata de personas con mucho tiempo libre, 15 horas al día, y que no realizan ningún trabajo, 0,10 horas diarias, y ningún trabajo doméstico. Se sitúan en el cuadrante inferior izquierdo de los ejes opuesto, sobre el primer eje (trabajo/tiempo libre), a los grupos 2 y 3 y, sobre el segundo (trabajo doméstico), al 1. Tiene una gran simetría radial con el grupo 3. No llega a un quinto de la población ${ }^{4}$.

Tipo 5: Está caracterizado por ser un grupo muy poco numeroso al límite de su significación representativa. Gran dedicación al trabajo productivo, 12 horas diarias, y sin ningún resquicio de tiempo libre, 2 horas diarias, y poco trabajo doméstico. Está situado completamente a la derecha del primer eje ligeramente escorados hacia abaj $0^{5}$.

Tipo 6: Tipo no demasiado numeroso. Formado por personas que realizan una gran superdedicación al trabajo doméstico, muy poca dedicación al trabajo productivo, 1,8 horas, y poco tiempo libre, 2 horas. Está situado sobre el segundo eje en su parte superior ${ }^{6}$.

La clasificación de los tipos de modos de vida cotidiana es casi perfecta desde el punto de vista de su simetría: cuatro grupos en los cuatro cuadrantes, dos a dos simétricos radial mente y otros dos grupos mucho menores que son extrapolaciones de algunas características de los primeros. El poder clasificador del soporte temporal solamente a partir de la articulación de ámbitos es manifiesto. Pero, tal y como nos lo planteábamos al principio, el segundo objetivo corresponde a si se puede intuir y predecir la connotación social de cada tipo.

1. 1.431 individuos (29\%). Primer eje: $x=-0,84$. Segundo eje: $y=0,44$

2. 1.904 individuos (36\%). Primer eje: $x=0,81$. Segundo eje: $y=-0,47$

3. 495 individuos $(9,7 \%)$. Primer eje: $x=1,05$. Segundo eje: $y=0,71$

4. 855 individuos $(17,7 \%)$. Primer eje: $x=-1,15$. Segundo eje: $y=-0,98$.

5. 79 individuos (1,6\%). Primer eje: $x=2,34$. Segundo eje: $y=-0,36$.

6. 262 individuos $(5,1 \%)$. Primer eje: $x=-0,13$. Segundo eje: $y=2,90$. 


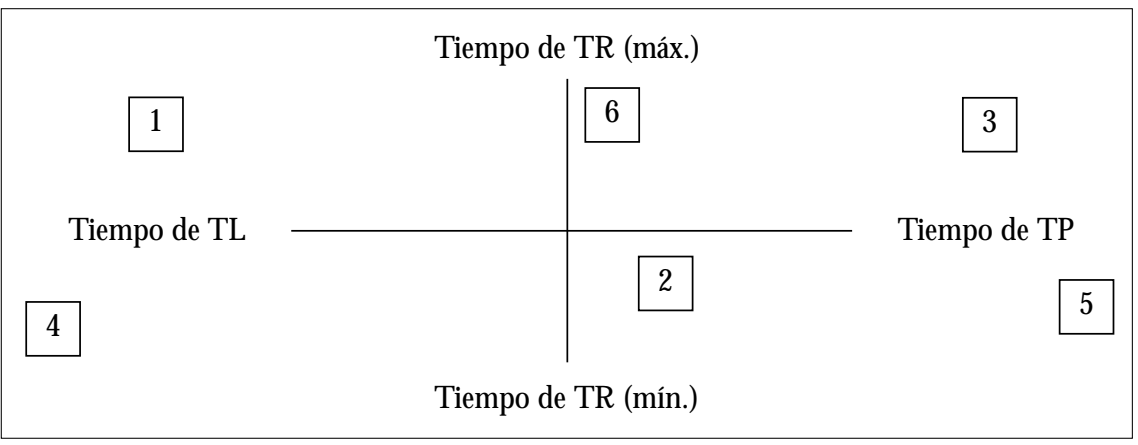

Gráfico 1. Gráfico de posiciones de los tipos en las tendencias

¿La simetría del tiempo estructurará la realidad social? ¿Será así el tiempo un buen predictor social? Si es así privará la hipótesis de correspondencia que augurábamos. Pero la cuestión permanece: iqué es prioritario o primigenio, la configuración de los grupos sociales a partir de la articulación de actividades, de relaciones, de «ocupaciones» de espacios y tiempos o la que proviene de indicadores atributivos que reflejen dimensiones más contextuales?

\subsubsection{La connotación social de los tipos de modos de vida encontrados o la inci-} dencia de las variables de categorización social

Se trata ahora de descubrir la identidad de los tipos de modos de vida cotidiana a partir de los factores de estructuración social. Precisamente estas consideraciones están en el centro de la confrontación metodológica empleada. Seguiremos un camino más descriptivo de perfiles. Básicamente el análisis está hecho a partir de la descripción bivariada confrontando la variable tipológica que acabamos de construir con las variables llamadas «de identificación» 0 «de estructuración social». D aremos una descripción sucinta. Veamos los resultados.

Tipo 1. Son personas con poca dedicación al trabajo productivo y, por consiguiente, con mucho tiempo libre y mucho tiempo empleado en el trabajo doméstico. Veamos su caracterización social, que por lo demás es completamente esperable. Con relación a este tipo se sitúan personas:

M ás próximasa

+56 años

Mujeres

$\mathrm{H}$ ogares unipersonales

Parejas sin hijos en casa

Ingresos medios-bajos o bajos o sin ingresos

CSE baja

Sin estudios o con estudios bajos

$O$ rigen individual: fuera de $C$ ataluña

La congruencia es pal pable.
M ás al ejadas u opuestas a

20-45 años

$\mathrm{H}$ ombres

Ingresos al tos o medios-altos

CSE media y alta

Estudios secundarios o universitarios $O$ rigen familiar: todos de C ataluña 
Tipo 2. Se trata de personas con gran dedicación al trabajo, poco al trabajo doméstico y poco tiempo libre disponible. Veamos su caracterización social previsible. Con relación a este tipo se sitúan personas:

M áspróximas a

20-45 años

Hombres

Pareja con hijos

Ingresos medios-altos o altos

CSE media o alta

Estudios secundarios o universitarios

O rigen individual catalán
M ásalejadas u opuestasa

56-65 años

Mujeres

Parejas sin hijos u hogares unipersonales Ingresos bajos, medios o medios-bajos

CSE baja o sin clasificar

Sin estudios o primarios

O rigen individual o familiar fuera de

Cataluña

D e nuevo, la correspondencia es visible.

Tipo 3. Está constituido por personas con dedicación al trabajo, poco tiempo libre y elevado tiempo dedicado al trabajo doméstico. Veamos quienes son por sus atributos sociales. Con relación a este tipo se sitúan personas:

M ás próximasa

26-55 años

Mujeres

Parejas con hijos

Ingresos medios-altos

CSE media o alta

Estudios primarios o universitarios

$M$ atrimonio mixto
$M$ ásalejadas u opuestasa

20-26, más de 56 años

$\mathrm{H}$ ombres

Parejas sin hijos u hogares unipersonales

Sin ingresos y bajos

CSE baja o sin clasificar

Sin estudios o secundarios

0 rigen familiar: todos fuera de $\mathrm{C}$ ataluña

La clasificación por tipos también separa socialmente.

Tipo 4. Se caracteriza por contener personas con mucho tiempo libre, que no realizan ningún trabajo productivo ni tampoco doméstico. Podemos suponer fácilmente de qué personas se trata. C on relación a este tipo se sitúan personas:

M áspróximas a

$20-26$ años o más de 56
Hombres
Pareja sin hijos en casa
Ingresos bajos

CSE sin clasificar

Sin estudios
Másalejadas u opuestasa
26-55 años
Mujeres
Pareja con hijos u hogares
Ingresos medios-altos
CSE media y alta
Estudios primarios o universitarios

O rigen familiar: todos fuera de C ataluña

La tipología de articulación de diferentes ámbitos es aquí también un buen clasificador social. 
Tipo 5. Está compuesto por personas con gran superdedicacion al trabajo, sin ningún resquicio de tiempo libre y con poco trabajo doméstico. No es difícil adivinar cuáles son sus características sociales. Con relación a este tipo se sitúan personas:

M ás proximas a

46-55 años pero sin excesiva asociación Hombres

Ingresos medios pero sin

excesiva asociación

Más bien de C SE media
M ás alejadasu opuestas a

M ás de 66 años pero sin fuerte asociación Mujeres

Ingresos bajos pero sin fuerte asociación

M ás bien de CSE «sin clasificar» pero de débil asociación

Es un tipo caracterizado socialmente aunque no de fuerte intensidad.

Tipo 6. Recordemos sus características, personas que realizan una gran superdedicacion al trabajo doméstico, dedicación media al trabajo productivo y poco tiempo libre ( 2 horas). Podemos suponer de quien se trata. Con relación a este tipo se sitúan personas.

M ás próximasa

46-55 años

Mujeres

$\mathrm{H}$ ogares unipersonales o monoparentales

Ingresos bajos/sin datos o sin ingresos

CSE baja o sin clasificar

Sin estudios

Origen individual de fuera de C ataluña
Más alejadasu opuestas a

20-26 años

Hombres

Ingresos medios-altos

CSE alta

Estudios secundarios o universitarios

\section{Reflexiones para unas conclusiones}

La idea de fondo que subyace en estas reflexiones es la siguiente: ¿no serán las prácticas (en nuestro caso actividades en tres ámbitos) tal y como se dan y se desarrollan en la vida cotidiana, con sus idas y venidas, sus lugares y sus tiempos y sus relaciones lo que constituye la realidad social, las categorías, los grupos, es decir, la estructura de la sociedad? ¿No será más bien que por el hecho de que la gente hace cosas parecidas o diferentes, se ve en los mismos o en diferentes contextos espaciales, mantiene idénticas, casi idénticas, 0 inexistentes relaciones, etc., son asignables a posiciones y grupos sociales y no debido al hecho de pertenecer o poseer una serie de cualidades 0 atributos inherentes? ¿No será acaso lo que se hace, se sucede y se relaciona lo que realmente crea la realidad social, y por ende los grupos sociales, siendo las propiedades, las cualidades, los atributos, etc. de los individuos o grupos redundantes o quizás espúreos? La virtud que tiene una metodología como la propuesta y desarrollada en el análisis está no sólo en la posi bilidad que ofrece de confirmar la justeza de las categorías que correspoden a los análisis macros, sino también la de poder hacer surgir grupos no previstos por dichas categorías. 
La arquitectura diaria, en el nivel sencillo que ha sido tratada y entre los ámbitos elegidos, es un descriptor social bueno y ajustado. Las dimensiones que emergen y los grupos que se forman guardan correlato con los factores y categorías con que habitualmente se trata de explicar y clasificar las real idades sociales. El resultado no es sorprendente aunque podría haberlo sido; incluso se ha de estar abierto a tal posibilidad. Si las prácticas propuestas hubieran sido más amplias, es decir, los ámbitos y la recogida de información más ad hoc para este objetivo, podrían haber surgido fenómenos o grupos más marginales o emergentes y, a partir de ahí, la aparición de alguna incoherencia, diferencia o contradición con los grupos más macrosociales, pero este proceso de análisis, y es lo interesante, podría haberlo auscultado. Así pues, los grupos que se forman por esta vía de análisis y conceptualización, más cercana a la realidad de la vida social de la gente tal y como se produce, manifiesta una buena y adecuada correspondencia con los que habitualmente clasificamos a la sociedad. Los modos de vida se proyectan en un espacio de variables clásicas: grupos de sexo, edad, ocupación, categorías socioeconómicas, etc.

El tiempo tiene así una función importante como distribuidor social de prácticas y como clasificador social. Los tipos o modos de vida cotidiana surgen de la articulación de dichos ámbitos a través del tiempo que se emplea en la realización de los mismos. Todo nos habla, pues, de la capacidad clasificadora y predictora, socialmente hablando, del tiempo.

Por encima y por debajo de nuestros model os y esquemas interpretativos la vida social se hace y nosotros podemos, por el análisis, dar una visión objetiva y válida de la misma, pero también puede ser deformada y desfigurada: sesgos sistemáticos 0 al eatorios, prejuicios en nuestra empresa de esquematizar, modelizar, tomar rasgos pertinentes, etc. Con las limitaciones que los datos nos imponían hemos tratado de utilizar un proceso de distribución de actividades en el tiempo. El resultado es la emergencia de líneas discriminantes, identidades inducidas, modos de vida y grupos de población correspondientes. Lo que resulta es que se consiguen formas pautadas de la vida social sin necesidad de pasar previamente por atributos generales o categorías macro preconstruidas y preestablecidas que son redundantes. Por añadidura ha resultado un apreciable parangón entre los grupos que «naturalmente» han emergido y las clásicas clasificaciones sociales.

\section{Bibliografía}

AYm ARD, M .; Grign on, A.; SABBAn, F. (1993a). «A la recherche du temps social». En Aym ARD, M .; GRIGn ON , A.; SABban, F. Le temps de manger. Alimentation, emploi du temps et rythmes sociaux. París: Éditions de la M aison des sciences de l'homme.

BERTEAUX, D . (1983). «Vie quotidienne ou modes de vie?». Revue suisse de Sociologie, 1: 67-84.

Bou Rdieu, P. (1963). «La Société traditionnelle». Sociologie du Travail, V: 24-44. Costanto pulou, C. (1988). «La gestión social del tiempo libre». En M ercure, D.; Wallem ACQ, A. Les temps sociaux. París: D e Boeck. 
ElIAS, N . (1985). Time: An Essay. Oxford: Blackwell.

Grossin (1974). LesTemps de la viequotidienne. París: Albin Michel.

JaVEAU, C. (1980). «Sur le concept de vie quotidienne et sa sociologie». Cahiers Internationaux de Sociologie, LXVIII: 31-45.

- (1983). «a sociologie du quotidienne: paradigme et enjeu». Revuesuisse de Sociologie, 1: 21-36.

LaLIVE d'Epinay, C. (1988). «El tiempo y el hombre comtemporáneo». En M ercure, D .; W Allem ACQ , A. Les temps sociaux. París: De Boeck.

LEACH, E.R. (1961). «Two Essays C oncerning the Symbolic Representation of Time». Rethinking Anthropology. Londres: University of London, The Athlone Press.

Mercure, D .; W allem ACQ , A. (1988). Les temps sociaux. París: D e Boeck.

REZSO HAZY, R. (1988). «Proyecto, previsión, memoria: concepto sociólogico e histórico». En M ercure, D.; W Allem ACQ, A. Les temps sociaux. París: De Boeck.

RaINER Zoll (1992). N ouvel individualisme et solidarité quotidienne. París: Éditions Kimé.

Rossi, A.S. (ed.) (1985). Gender and the Life Course. N ueva York: Aldine Publ.

SAM UEL, N icole (1984). Le temps libre: un temps social. París: Librairie des M éridiens. So Ro KIN, P.A. (1936). «s Accurate Social Planning Possible?». American Sociological Review, 1: 12-38.

SOROKIN, P.A.; M ERTON, R.K. (1937). «Social Time; A M ethodological and Functional Aanalysis». The American Journal of Sociology. 42: 615-629.

TABBON I, Simonetta (1986). La rappresentazione sociale del tempo. M ilán: Franco Angeli. W ALLACE, M. (1977). «Future Time Perspective in Schizophrenia». Journal of Abnormal and Social Psychology, 52: 240-245.

Wallem AC, A. (1988). «Los tiempos sociales, un pleonasmo. Ejercicio de estilo». En M ercure, D.; W allem AcQ, A. Lestemps sociaux. París: De Boeck. 